\title{
Children with cerebral palsy exhibit greater and more regular postural sway than typically developing children
}

\author{
Stella F. Donker · Annick Ledebt · Melvyn Roerdink • \\ Geert J. P. Savelsbergh · Peter J. Beek
}

Received: 21 March 2007 / Accepted: 13 August 2007/Published online: 2 October 2007

(C) Springer-Verlag 2007

\begin{abstract}
Following recent advances in the analysis of centre-of-pressure (COP) recordings, we examined the structure of COP trajectories in ten children (nine in the analyses) with cerebral palsy (CP) and nine typically developing (TD) children while standing quietly with eyes open (EO) and eyes closed (EC) and with concurrent visual COP feedback (FB). In particular, we quantified COP trajectories in terms of both the amount and regularity of sway. We hypothesised that: (1) compared to TD children, $\mathrm{CP}$ children exhibit a greater amount of sway and more regular sway and (2) concurrent visual feedback (creating an external functional context for postural control, inducing a more external focus of attention) decreases both the amount of sway and sway regularity in TD and CP children alike, while closing the eyes has opposite effects. The data were largely in agreement with both hypotheses. Compared to TD children, the amount of sway tended to be larger in $\mathrm{CP}$ children, while sway was more regular. Furthermore, the presence of concurrent visual feedback resulted in less
\end{abstract}

S. F. Donker · A. Ledebt $(\bowtie) \cdot$ M. Roerdink ·

G. J. P. Savelsbergh · P. J. Beek

Research Institute MOVE, Faculty of Human Movement

Sciences, VU University Amsterdam, Van der Boechorststraat 9,

1081 BT Amsterdam, The Netherlands

e-mail: a_ledebt@fbw.vu.nl

\section{S. F. Donker}

Department of Otorhinolaryngology,

VU University Medical Centre, Amsterdam, The Netherlands

e-mail: s.f.donker@uu.nl

G. J. P. Savelsbergh

Research Institute for Biophysical and Clinical Research into Human Movement, Manchester Metropolitan University, Manchester, UK regular sway compared to the EO and EC conditions. This effect was less pronounced in the $\mathrm{CP}$ group where posturograms were most regular in the EO condition rather than in the EC condition, as in the control group. Nonetheless, we concluded that $\mathrm{CP}$ children might benefit from therapies involving postural tasks with an external functional context for postural control.

Keywords Cerebral palsy · Postural control · Attention · Centre-of-pressure trajectories $\cdot$ Regularity

\section{Introduction}

Poor postural control has been suggested to underlie the delays and deviations in motor skill acquisition and development observed in children with cerebral palsy (CP) (e.g., Berger et al. 1984; Liao et al. 1997; Liao and Hwang 2003). In particular, children with CP encounter problems during static upright standing in altered sensory environments (Cherng et al. 1999; Rose et al. 2002) and when rapid weight shifts during standing are required, either in gait initiation (Stackhouse et al. 2007) or in reaction to external perturbations (Nashner et al. 1983; Woollacott and Burtner 1996).

Postural control is often assessed by means of posturography, that is, the quantitative analysis of centre-ofpressure (COP) trajectories as measured with a force platform. Several descriptive statistics of the COP time series, which are often derived by averaging out the assumed noisy or random character of postural sway, have been shown to change with various motor, sensory and cognitive processes involved in the control of standing (e.g., Horak 2006; Woollacott and Shumway-Cook 2002). In the past two decades, however, new concepts and 
methods for studying postural control have been introduced based on the assumption that the act of maintaining an erect posture may be viewed as a stochastic process (Collins and De Luca 1993; Newell et al. 1997; Riley et al. 1999; Frank et al. 2001). Based on these insights, new measures have been developed for examining the timevarying or dynamical characteristics of COP trajectories. These dynamical measures may be more informative with regard to changes in postural control with task constraints, aging or pathology than the more global summary statistics that have been typically employed for this purpose, or at least provide important additional information (see Baratto et al. 2002; Raymakers et al. 2005; Roerdink et al. 2006).

Recent research has suggested that the regularity of COP trajectories is a particularly revealing and theoretically important dynamical measure. As a case in point, Roerdink et al. (2006) found that COP trajectories were more regular (as indexed by reduced sample entropy) in stroke patients than in healthy elderly while COP trajectories became progressively less regular with recovery. These findings may be viewed in line with the notion of pathological regularity versus healthy complexity (e.g., Goldberger 1996, 1997). Moreover, when performing a secondary cognitive task COP trajectories became less regular (Roerdink et al. 2006). The latter finding was replicated in young healthy adults, albeit only in the more attentiondemanding eyes closed condition (Donker et al. 2007). Based on these results, a direct relation was proposed between the regularity of COP time series and the amount of attention invested in postural control (or, inversely, between COP regularity and the degree of automatism of postural control; see Donker et al. 2007; Roerdink et al. 2006). In particular, the introduction of a secondary cognitive task withdraws attention from the regulation of posture (i.e., towards the performance of the attentiondemanding secondary task), resulting in less regular COP trajectories. This interpretation is in line with the claim and corresponding findings of Wulf and colleagues that adopting an external focus of attention is beneficial for the performance and learning of motor skills, whereas an internal focus of attention (e.g., directing attention to one's own body and bodily movements) can be detrimental as it may disrupt the 'automatisms' with which well-learned skills are normally performed (for a review see Wulf and Prinz 2001; see also Wulf et al. 2001; McNevin and Wulf 2002; McNevin et al. 2003).

When applied to CP children this insight may imply that their postural control may be improved by means of instructions or task conditions that direct their attention away from the regulation of posture itself to its consequences in the world. Providing $\mathrm{CP}$ children with concurrent visual feedback about postural sway may help create a more external focus of attention (i.e., the external consequences of postural sway become readily apparent via visual cursor movements). Visual feedback of postural sway provides a functional context for postural control (e.g., minimise cursor movements), which is likely to enhance the performance of the primary task (cf., Lin et al. 1998; Van der Weel et al 1991; Volman et al. 2002). For example, Volman et al. (2002) showed that the quality of reaching movements of the affected arm in children with spastic hemiparesis could be enhanced when reaching to press a light switch to turn on a light (task with functional context) compared to reaching to a marker (nonfunctional task). We therefore expected that postural control could benefit from postural visual feedback as it constitutes a task with a functional external context for postural control, inducing a more external focus of attention. Although postural visual feedback is sometimes used by physical therapists to train postural control and steadiness (i.e., the ability to maintain a given posture with minimal sway, Nichols 1997), evidence for this practice is limited (e.g., Rougier 2003; Ledebt et al. 2005). The recent conceptual and methodological developments in the analysis of COP trajectories alluded to in the preceding provide an alternative approach for studying the consequences of concurrent visual feedback for postural control and thus of its potential for possible therapeutic applications.

Motivated by this combination of theoretical and practical considerations, the aim of the present study was (1) to compare postural sway fluctuations of children with $\mathrm{CP}$ with that of typically developing (TD) children and (2) to investigate the effect of visual information on postural sway in CP children and in TD children. For this purpose, the availability of visual information was manipulated relative to standing with eyes open (EO condition) by means of visual deprivation (standing with eyes closed, EC condition) and external provision of COP feedback (FB condition). Two kinds of measures were used to analyse the COP trajectories recorded during those conditions, the one scale-dependent and the other scaleindependent. The conventional, scale-dependent measures pertained to the 'amount of sway', whereas the more recent scale-independent measures pertained to the 'regularity of sway' (see Methods section for details). We hypothesised that (1) compared to TD children, CP children exhibit a greater amount of sway and more regular sway and (2) concurrent visual feedback (providing a functional external context for postural control, creating a more external focus of attention) decreases both the amount of sway and sway regularity in TD and CP children alike, while closing the eyes (increasing task difficulty through deprivation of visual information, promoting a less automatic mode of postural control) has opposite effects. 


\section{Methods}

Participants

Ten children with congenital hemiplegia or cerebral palsy (CP children; six boys, four girls; mean age $=7$ years, range $=5-11$ years), reduced to nine in the analyses (see below) and nine typically developing children (TD children; five boys, four girls; mean age $=8$ years, range $=5-11$ years) without known motor impairments or movement-related disorders volunteered to participate in the experiment. All children and their parents gave their informed consent prior to participation. The experiment was carried out in compliance with the Helsinki Declaration and was endorsed by the VU University Medical Centre Amsterdam.

\section{Procedure and apparatus}

Children stood upright on a $1 \mathrm{~m} \times 1 \mathrm{~m}$ custom-made strain gauge force plate ${ }^{1}$ with their arms hanging relaxed alongside the body. Their feet were placed parallel to the anterior-posterior axis of the force plate and the distance between the feet corresponded to the width of the pelvis. Children wore their own shoes, including ankle-foot ortheses and/or insoles to correct for leg-length discrepancies.

Children participated in three conditions that were presented in random order: standing upright with eyes open (EO condition), standing upright with eyes closed (EC condition) and standing upright with visual COP feedback (FB condition). As explained, the FB condition was introduced to provide an external functional context for postural control, creating a more external focus of attention. To this end, a $2.5 \mathrm{~m} \times 2.5 \mathrm{~m}$ vertical screen was placed in front of the child at a distance of $1.3 \mathrm{~m}$. On this screen the force plate was depicted as a $40 \mathrm{~cm} \times 40 \mathrm{~cm}$ square onto which the COP was projected as a red dot. The children were asked to keep this dot within the target area, which consisted of a $4 \mathrm{~cm} \times 4 \mathrm{~cm}$ square. Deprivation of visual postural sway information was effectuated by blindfolding the children in the EC condition. Trial duration was $60 \mathrm{~s}$. Participants performed each condition once, with the proviso that, as part of a separate study, five CP children performed each condition twice (the first trial was used for further analyses). Because in the latter set of recordings trial duration was $30 \mathrm{~s}$, the first 3,000 samples of all trials were used in the analyses to avoid differences in

\footnotetext{
${ }^{1}$ Analog force signals were digitised into a 12-bit signal at a sampling rate of $100 \mathrm{~Hz}$ by an AD converter (NI PCi 6040E, National Instruments, Austin, TX, USA)
}

the reliability of the dependent measures. One $\mathrm{CP}$ child did not want to be blindfolded and was therefore excluded from further analyses, which rendered the number of $\mathrm{CP}$ children equal to the number of control subjects.

\section{Data analysis}

Prior to all analyses, the mean was subtracted from mediolateral and anterioposterior COP trajectories, which transformed the original time series into $x$ and $y$ time series, respectively. Subsequently, $x$ and $y$ time series were bidirectionally filtered (second-order low-pass Butterworth filter, cut-off frequency $12.5 \mathrm{~Hz}$ ) and the resultant distance time series $r$ was constructed using $r_{i}=\sqrt{x_{i}^{2}+y_{i}^{2}}$ (see Prieto et al. 1996), with $i=1,2,3, \ldots, N$ and $N$ indicating the total number of analysed samples in the COP time series (i.e., 3,000 samples).

The 'amount of sway' was quantified by means of two conventional, scale-dependent measures (see Prieto et al. 1996). First, the average COP distance to the origin of the mean-centred posturogram was determined by taking the mean of the $r$ time series (i.e., mean amplitude $r_{\text {mean }}$ in $\mathrm{mm})$. Second, sway path length (SP in $\mathrm{mm}$ ) was determined by taking the sum of the distances between consecutive points in the conventional posturogram, using $\mathrm{SP}=\sum_{i=1}^{N-1} \sqrt{\left(x_{i+1}-x_{i}\right)^{2}+\left(y_{i+1}-y_{i}\right)^{2}}$.

To examine the structure of COP trajectories in more detail, independent of its size or scale, $x$ and $y$ were normalised to unit variance by dividing those time series by their respective standard deviations $\sigma_{x}$ and $\sigma_{y}$, resulting in a normalised posturogram. Subsequently, two scale-independent COP measures were quantified. First, the sway path of the normalised posturogram $\left(\mathrm{SP}_{n}\right)$ was determined in a similar manner as described above for the conventional posturogram. Because the posturograms were normalised to its size, differences in $\mathrm{SP}_{n}$ could only be the result of changes in the structure of the posturogram, with a larger $\mathrm{SP}_{n}$ indicating a larger amount of 'twisting and turning' or 'curviness' in the COP trajectory (cf., Donker et al. 2007). Second, COP regularity was quantified by calculating the sample entropy (cf., Richman and Moorman 2000; Lake et al. 2002; see also Roerdink et al. 2006), which is a slightly improved version of the approximate entropy (cf., Pincus 1991). The sample entropy (SEn) is the negative natural logarithm of an estimate of the conditional probability that subseries (epochs) of length $m$ (in our case, $m=3$ ) that match pointwise within a specific tolerance also match at the next point (for a more formal and detailed explanation see Richman and Moorman 2000; Lake et al. 2002). In other words, smaller SEn values imply more regular COP time series, that is, a greater likelihood that 
Table 1 Main and interaction effects of group (between-subject factor, two levels: CP and TD children) and condition (within-subject factor, three levels: EO, EC and FB) on mean sway amplitude $\left(r_{\text {mean }}\right)$, sway path of the conventional posturogram (SP), sway path of the normalised posturogram $\left(\mathrm{SP}_{n}\right)$ and sample entropy (SEn)

\begin{tabular}{|c|c|c|c|c|c|c|c|c|c|}
\hline & \multicolumn{3}{|l|}{ Group } & \multicolumn{3}{|c|}{ Condition } & \multicolumn{3}{|c|}{ Interaction } \\
\hline & $F_{(1,16)}^{\mathrm{a}}$ & $P$-value & $\varepsilon_{p}^{2}$ & $F_{(2,32)}^{\mathrm{a}}$ & $P$-value & $\varepsilon_{p}^{2}$ & $F_{(2,32)}^{\mathrm{a}}$ & $P$-value & $\varepsilon_{p}^{2}$ \\
\hline$r_{\text {mean }}$ & 4.01 & 0.062 & 0.20 & 1.35 & ns & 0.08 & 1.11 & ns & 0.06 \\
\hline SP & 1.86 & ns & 0.10 & 0.13 & ns & 0.01 & 0.13 & ns & 0.01 \\
\hline $\mathrm{SP}_{n}$ & 11.11 & $<0.005$ & 0.41 & 5.12 & $<0.02$ & 0.24 & 2.04 & ns & 0.11 \\
\hline SEn & 13.49 & $<0.005$ & 0.46 & 5.53 & $<0.01$ & 0.26 & 3.23 & 0.056 & 0.17 \\
\hline
\end{tabular}

$n s$ not significant

${ }^{a}$ In case the assumption of sphericity was violated, the number of degrees of freedom was adjusted using the Huynh-Feldt method (corrected degrees of freedom are not listed here)

sets of matching epochs in a time series will be followed by another match within a certain tolerance. On the other hand, highly irregular COP time series are characterised by the fact that sets of matching epochs tend to be followed by data samples of different values, resulting in larger SEn estimates. In the present study, SEn was quantified from the $r$ time series, which was first normalised to unit variance. To optimise the choice of the tolerance for a given $m$ (see also Roerdink et al. 2006) we applied the approach of Lake et al. (2002) and selected the median value of the optimal tolerance over all trials (in our case, 0.05). The code used for calculating sample entropy was obtained from PhysioNet ${ }^{2}$ (Goldberger et al. 2000).

To rule out the possibility that COP trajectories exhibited regular or deterministic patterns by chance, we compared the SEn estimates obtained for the COP trajectories to those obtained for surrogate data (cf., Theiler et al. 1992). We used time-randomisation to preserve the probability distribution of the data (e.g., mean, variance) while destroying the temporal correlations in the data, resulting in very large values for SEn. In contrast, phase-randomisation randomises the phases of the data in the Fourier domain but preserves the probability distribution of the data and its spectral power distribution (cf., Kantz and Schreiber 2004). Consequently, estimates of SEn for phase-randomised COP data should be somewhat increased, although less pronounced as for the time-randomised COP data.

\section{Statistics}

A repeated-measures analysis of variance (ANOVA) was conducted on all dependent variables with group as between-subject factor (two levels: $\mathrm{CP}$ and TD children) and condition as within-subject factor (three levels: EO, EC and FB conditions). The effect of randomisation was evaluated using a one-way repeated measures ANOVA

$\overline{2}$ http://www.physionet.org/. with the factor randomisation (three levels: original, phaserandomised and time-randomised time series), including each individual time series as a sample. Effect sizes for main and interaction effects are reported as partial eta squared $\left(\varepsilon_{p}^{2}\right)$. Besides significant effects $(P<0.05)$, also tendencies towards significance $(P<0.10)$ are reported in view of the limited sample size. Paired-samples $t$-tests were used for post-hoc analyses of significant condition or randomisation effects. The analyses were performed using SPSS (SPSS, Inc., Chicago IL, USA).

\section{Results}

The results ( $F, P$ and $\varepsilon_{p}^{2}$ values) of the group by condition ANOVA for the dependent COP variables are presented in Table 1. In Fig. 1, the group by condition effects are summarised for all dependent measures. CP children's sway amplitude was greater than that of TD children (i.e., $r_{\text {mean }}=12.8 \mathrm{~mm}$ and $5.7 \mathrm{~mm}$, respectively, although this difference was strictly speaking not significant, $P=0.062$ ). Neither a significant main effect of condition nor a significant group by condition interaction effect was observed for $r_{\text {mean }}$. Furthermore, no significant main or interaction effects were observed for the sway path in the conventional posturogram (SP; mean $1,367 \mathrm{~mm}$, standard error $108 \mathrm{~mm}$ ), whereas the sway path in the normalised posturogram $\left(\mathrm{SP}_{n}\right)$ differed significantly between groups and conditions in the absence of a significant interaction (see Table 1). Specifically, $\mathrm{SP}_{n}$ was significantly larger for the TD children (328) than for the CP children (212). Moreover, post-hoc analysis revealed that concurrent visual feedback significantly increased $\mathrm{SP}_{n}$ (310) compared to both standing with eyes open $\left(\mathrm{SP}_{n}=259: t_{(17)}=2.41\right.$, $P<0.05)$ and standing with eyes closed $\left(\mathrm{SP}_{n}=243\right.$ : $\left.t_{(17)}=2.49, P<0.05\right)$.

As can be seen in Table 1, the main effects of group and condition were both significant for sample entropy, while the group by condition interaction tended towards 
Fig. 1 Effects of group $(C P$ and $T D$ children, represented by black and grey bars, respectively) and condition ( $E O$, $E C$ and $F B$ ) on the 'amount of sway' (upper panels, mean sway amplitude $r_{\text {mean }}$ and sway path of the conventional posturogram $S P$ ) and on the 'regularity of sway' (lower panels, sway path of the normalised posturogram $\mathrm{SP}_{n}$ and sample entropy SEn; note that lower SEn values imply more regular posturograms). Error bars represent the standard error
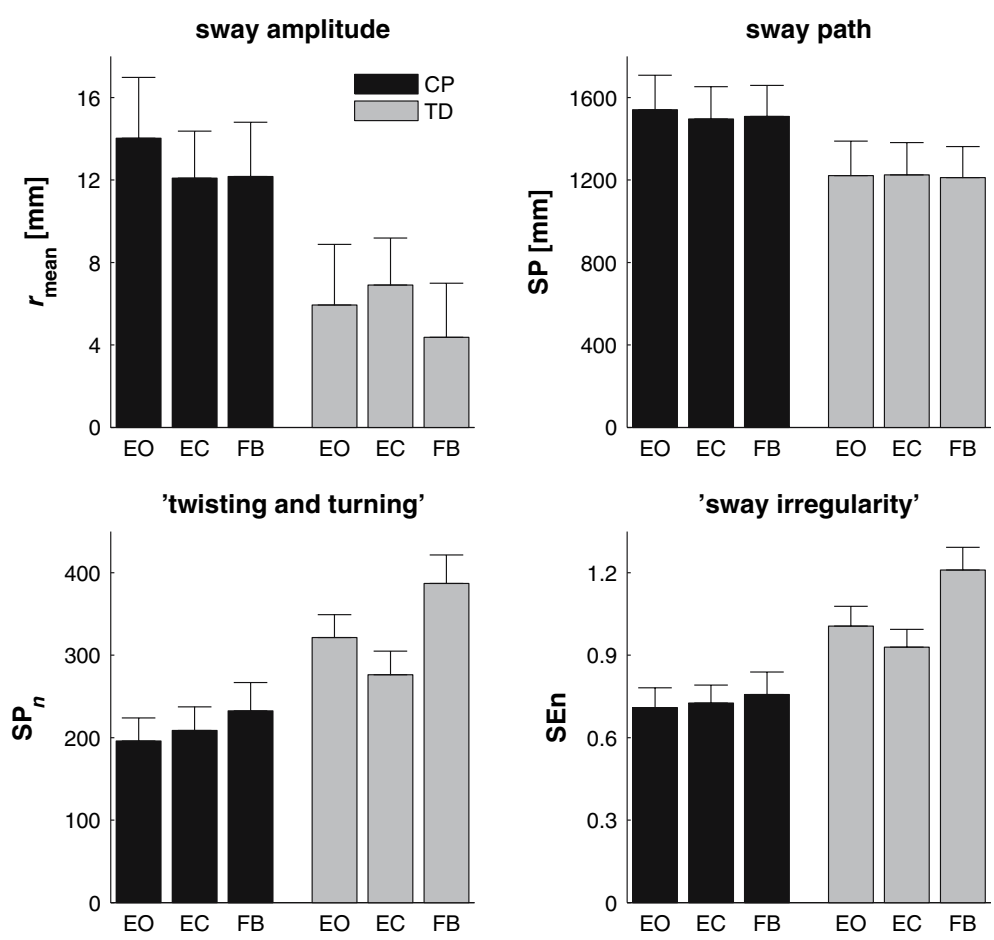

significance $(P=0.056)$. SEn was significantly larger for the TD children (1.01) than for the CP children (0.71). Moreover, post-hoc analysis revealed that the FB condition significantly increased SEn (0.98) compared to both standing with eyes open $\left(\mathrm{SEn}=0.86: t_{(17)}=2.37\right.$, $P<0.05)$ and standing with eyes closed $(\mathrm{SEn}=0.83$ : $\left.t_{(17)}=2.42, P<0.05\right)$. The observed tendency of the group by condition interaction towards significance (see Fig. 1, lower right panel) may be due to the fact that for $\mathrm{CP}$ children SEn increased gradually over EO, EC and FB conditions (0.71, 0.73 and 0.76 , respectively), whereas in the TD children SEn first reduced in the EC condition (0.93) and then increased for the FB condition (1.21) as compared to standing with eyes open (1.01).

The surrogate analysis revealed that SEn differed significantly with randomisation $\left(F_{(2,106)}=2915.90, P<\right.$ $\left.0.001, \varepsilon_{p}^{2}=0.98\right)$. Post-hoc $t$-tests indicated that SEn differed significantly over all comparisons (all $t_{(53)}>7.2$, all $P<0.001$ ), indicating that the regular or deterministic patterns observed in the original data did not occur by chance. SEn was on average (standard error) 0.89 (0.04), $1.06(0.04)$ and $3.44(0.02)$ for original, phase-randomised and time-randomised COP data, respectively.

\section{Discussion}

In the present study we compared posturograms of children with $\mathrm{CP}$ with those of TD children under three conditions. Across conditions, we expected the posturograms of $\mathrm{CP}$ children to reflect a greater amount of sway and more regular sway than those of TD children (Hypothesis 1). Furthermore, in line with the recently postulated relation between the amount of attention invested in posture and COP regularity, we expected that concurrent visual COP feedback, providing a functional external context for postural control leading to a more external focus of attention, would result in a decrease in both the amount of sway and sway regularity, whereas the EC condition, in which visual information is absent, would have opposite results (Hypothesis 2). The data were largely in agreement with both hypotheses, as will be discussed next. Furthermore, the finding that SEn estimates were significantly increased after time- and phase-randomisation of the original data ruled out the possibility that the regular or deterministic properties observed in the original data occurred by chance.

$\mathrm{CP}$ children exhibit greater and more regular sway than TD children

Posturogram characteristics of children with $\mathrm{CP}$ differed markedly from those of TD children (see Fig. 1). As expected (Hypothesis 1), the CP children exhibited a larger amount of sway than the TD children. In particular, a near significant $(P=0.062$, accompanied by a large effect size $)$ increment in mean sway amplitude $\left(r_{\text {mean }}\right)$ was observed in the $\mathrm{CP}$ children compared to that in the TD children (see Table 1 and Fig. 1). Although, strictly speaking, not significant due to the large interindividual variations, this 
effect suggests that 'postural steadiness' is reduced in $\mathrm{CP}$ children, consistent with previous results on balance control in CP children (e.g., Cherng et al. 1999; Ferdjallah et al. 2002; Liao and Hwang 2003; Rose et al. 2002). An interesting and unexpected finding was that the total COP excursion (i.e., SP) did not differ significantly between $\mathrm{CP}$ and TD children. In combination with the apparent increase in sway amplitude, this finding hints at the presence of marked differences in the dynamical structure of posturograms between $\mathrm{CP}$ and TD children. To expose those differences, we focused on the sway regularity using two scale-independent measures. Scale-independence was achieved by normalising the data to unit variance. As expected, sway path length of the normalised posturogram was significantly reduced in CP children, indicating less 'twisting and turning' in the posturogram (see also Donker et al. 2007). Relatedly, resultant COP trajectories of the CP children were more regular than those of the TD children, as indexed by significantly lower sample entropy values. Recently, increased regularity in COP trajectories has been reported for a variety of pathological conditions, including athletes with a sports-related cerebral concussion (Cavanaugh et al. 2005 using approximate entropy), patients with Parkinson's disease (Schmit et al. 2006 using recurrence quantification analysis) and stroke patients (Roerdink et al. 2006 using sample entropy). These findings are in line with the more general notion of 'dynamical diseases' (Glass and Mackey 1988; Belair et al. 1995) and the 'pathological regularity versus healthy complexity' hypothesis (Goldberger 1996, 1997; Goldberger et al. 2002; Kyriazis 2003; Lipsitz 2002), according to which less 'complex' or more 'regular' physiological time series reflect less effective physiological control. In a similar vein, the observed increased COP regularity in CP children may be viewed as the dynamical signature of poor postural control.

\section{Effect of visual information}

We further expected that the 'amount of sway' would be reduced in the presence of concurrent visual COP feedback, providing a supposedly beneficial functional external context for postural control (cf., Van der Weel et al. 1991; Volman et al. 2002; Wulf and Prinz 2001), as opposed to deprivation of visual information in standing with eyes closed. However, no significant main effects of condition were found for the scale-dependent COP measures (i.e., mean amplitude and sway path, see Table 1). This unexpected result is in line with some recent studies showing that the use of visual COP feedback to facilitate quiet standing has no effect on conventional COP parameters in TD children (Lebiedowska and Syczewska 2000) and healthy elderly and stroke patients (Dault et al. 2003) alike.
These findings suggest that participants may require some practice to benefit from visual COP feedback. This may explain why, in spite of the apparent lack of immediate effects, Ledebt et al. (2005) found that balance training with visual feedback improved CP children's quiet standing ability as well as their functional limits of stability as assessed by a weight-shifting task.

In contrast to the results obtained for the 'amount of sway' under the various conditions, the scale-independent measures pertaining to the 'regularity of sway' did show significant differences between the FB condition and the EO and EC conditions. This indicates that the dynamical structure of the posturogram must have been different in the FB condition compared to the EO and EC conditions, which was precisely what we anticipated when formulating our expectation that sway would be less regular in the FB condition (i.e., longer sway path of the normalised posturogram and higher sample entropy) and more regular in the EC condition (Hypothesis 2). Sway path of the normalised posturogram was indeed significantly longer with visual feedback than for standing with eyes open or closed, indicating more twisting and turning (see also Donker et al. 2007). In parallel, sample entropy increased with concurrent visual feedback compared to standing with eyes open and eyes closed. Thus, in spite of the fact that the 'amount of sway' did not change significantly over conditions, the observed changes in 'regularity of sway' were as expected: sway was more regular in the EC condition and less regular in the FB condition.

Our expectations concerning the 'regularity of sway' with conditions were derived from the proposed direct relation between the amount of attention invested in posture and the regularity of COP trajectories (cf., Donker et al. 2007; Roerdink et al. 2006). This interpretation is in line with the insight of Wulf and colleagues that adopting an external focus of attention, i.e., a focus on the consequences of one's movement in the external world, is beneficial for the performance and learning of motor skills (McNevin et al. 2003; McNevin and Wulf 2002; Wulf and Prinz 2001; Wulf et al. 2001). Specifically, we expected that providing $\mathrm{CP}$ children with concurrent visual feedback about postural sway would help shift the focus of attention to the external consequences of postural sway (i.e., cursor movements) by constituting a functional external context for postural control (e.g., minimise cursor movements). Providing a functional context for the task at hand generally enhances its performance (e.g., Lin et al. 1998; Van der Weel et al. 1991; Volman et al. 2002). In line with these notions, we expected that standing with concurrent visual feedback, a functional task inducing a more external focus of attention, would lead to less regular sway compared to the EO condition, whereas standing with eyes closed would lead to more regular sway in the EC condition compared to the EO condition because task difficulty 
increased due to the absence of visual information. This predicted pattern of results was only found for the TD children and not for the $\mathrm{CP}$ children (see lower right panel of Fig. 1). As it turned out, COP regularity in the $\mathrm{CP}$ children gradually decreased from the EO condition (most regular), via the EC condition, to the FB condition (least regular). As a result of this difference in group behaviour, a strong tendency towards a significant group by condition interaction on sample entropy was found. From this latter observation one could tentatively infer that CP children's posture actually improved when standing with eyes closed compared to standing with eyes open. In support of this somewhat counter-intuitive inference, Rose et al. (2002) showed that the percentage of $\mathrm{CP}$ children with abnormal sway characteristics did not differ between EO and EC conditions, while the number of sway path outliers even reduced when standing with eyes closed in that particular $\mathrm{CP}$ group. Following a similar line of interpretation, Newell et al. (1997) suggested that very young children may create self-induced perturbations when using visual information in controlling posture, a phenomenon which may be particularly prominent in $\mathrm{CP}$ children given that the development of proper neural control mechanisms is strongly delayed compared to TD children (viz., Forssberg 1999). From the opposite SEn results between CP and TD children with eyes open and eyes closed it is fair to conclude that postural control in CP children is organised differently than in TD children, especially with regard to the use of normal sway-induced visual information.

It has been suggested that creating an external focus of attention would allow for a more automatic control of movements (e.g., Donker et al. 2007; McNevin and Wulf 2002; Roerdink et al. 2006), which is often found to be more efficient (e.g., Blanchard et al. 2005; McNevin et al. 2003). Although no significant main effect of condition on mean amplitude was found, the finding that during quiet standing with visual COP feedback sample entropy increased (i.e., less regularity in the posturogram) in both TD and CP children supports this idea. Moreover, it corroborates the suggestion that visual COP feedback may be advantageous when seeking to improve postural control, although it must be emphasised again that it requires training (see Ledebt et al. 2005).

\section{Conclusion}

The findings of the present study indicate that postural control in $\mathrm{CP}$ children is deteriorated compared to TD children, most likely due to a slowed development of neural control mechanisms in $\mathrm{CP}$ children. Moreover, visual deprivation (EC condition) influenced sway characteristics differently in $\mathrm{CP}$ and $\mathrm{TD}$ children, testifying to the altered nature of postural control in CP children. In line with the proposed relation between the amount of attention invested in posture and COP regularity, we found in $\mathrm{CP}$ and TD children alike that, compared to the EO and EC condition, COP trajectories were less regular in the presence of visual COP feedback, corresponding to a functional context mediated external shift in the focus of attention. Future studies should examine the merits of adopting an external focus of attention to enhance postural control in $\mathrm{CP}$ children in training or therapy, for example by providing visual COP feedback leading to a posture specific functional task or by introducing an attention-demanding secondary cognitive task.

Acknowledgments The contribution of the third author was facilitated by the Netherlands Organisation for Health Research and Development (ZonMw, grant 1435.0004). The first author is now with the Department of Experimental Psychology of University of Utrecht.

\section{References}

Baratto L, Morasso P, Re C, Spada G (2002) A new look at posturographic analysis in the clinical context: sway-density vs. other parameterization techniques. Motor Control 6:246-270

Belair J, Glass L, An Der Heiden U, Milton J (1995) Dynamical disease: identification, temporal aspects and treatment strategies of human illness. Chaos 5:1-7

Berger W, Altenmueller E, Dietz V (1984) Normal and impaired development of children's gait. Hum Neurobiol 3:163-170

Blanchard Y, Carey S, Coffey J, Cohen A, Harris T, Michlik S, Pellecchia GL (2005) The influence of concurrent cognitive tasks on postural sway in children. Pediatr Phys Ther 17:189-193

Cavanaugh JT, Guskiewicz KM, Stergiou N (2005) A nonlinear dynamic approach for evaluating postural control: new directions for the management of sport-related cerebral concussion. Sports Med 35:935-950

Cherng RJ, Su FC, Chen JJ, Kuan TS (1999) Performance of static standing balance in children with spastic diplegic cerebral palsy under altered sensory environments. Am J Phys Med Rehabil 78:336-343

Collins JJ, De Luca CJ (1993) Open-loop and closed-loop control of posture: a random-walk analysis of center-of-pressure trajectories. Exp Brain Res 95:308-318

Dault MC, de Haart M, Geurts AC, Arts IM, Nienhuis B (2003) Effects of visual center of pressure feedback on postural control in young and elderly healthy adults and in stroke patients. Hum Mov Sci 22:221-236

Donker SF, Roerdink M, Greven AJ, Beek PJ (2007) Regularity of center-of-pressure trajectories depends on the amount of attention invested in postural control. Exp Brain Res 181:1-11

Ferdjallah M, Harris GF, Smith P, Wertsch JJ (2002) Analysis of postural control synergies during quiet standing in healthy children and children with cerebral palsy. Clin Biomech 17:203210

Forssberg H (1999) Neural control of human motor development. Curr Opin Neurobiol 9:676-682

Frank TD, Daffertshofer A, Beek PJ (2001) Multivariate OrnsteinUhlenbeck processes with mean-field dependent coefficients: application to postural sway. Phys Rev E 63:0011905/1-16

Glass L, Mackey MC (1988) From clocks to chaos: the rhythms of life. Princeton University Press, Princeton 
Goldberger AL (1996) Non-linear dynamics for clinicians: chaos theory, fractals, and complexity at the bedside. Lancet 347:1312-1314

Goldberger AL (1997) Fractal variability versus pathological periodicity: complexity loss and stereotypy in disease. Perspect Biol Med 40:543-561

Goldberger AL, Amaral LA, Glass L, Hausdorff JM, Ivanov PC, Mark RG, Mietus JE, Moody GB, Peng CK, Stanley HE (2000) PhysioBank, PhysioToolkit, and PhysioNet: components of a new research resource for complex physiologic signals. Circulation 101:E215-E220

Goldberger AL, Amaral LA, Hausdorff JM, Ivanov P, Peng CK, Stanley HE (2002) Fractal dynamics in physiology: alterations with disease and aging. Proc Natl Acad Sci USA 99(Suppl 1):2466-2472

Horak FB (2006) Postural orientation and equilibrium: what do we need to know about neural control of balance to prevent falls? Age Ageing 35(Suppl 2):ii7-ii11

Kantz H, Schreiber T (2004) Nonlinear time series analysis. Cambridge University Press, Cambridge

Kyriazis M (2003) Practical applications of chaos theory to the modulation of human ageing: nature prefers chaos to regularity. Biogerontology 4:75-90

Lake DE, Richman JS, Griffin MP, Moorman JR (2002) Sample entropy analysis of neonatal heart rate variability. Am J Physiol Regul Integr Comp Physiol 283:R789-R797

Lebiedowska MK, Syczewska M (2000) Invariant sway properties in children. Gait Posture 12:200-204

Ledebt A, Becher J, Kapper J, Rozendaal RM, Bakker R, Leenders IC, Savelsbergh GJ (2005) Balance training with visual feedback in children with hemiplegic cerebral palsy: effect on stance and gait. Motor Control 9:459-468

Liao HF, Hwang AW (2003) Relations of balance function and gross motor ability for children with cerebral palsy. Percept Mot Skills 96:1173-1184

Liao HF, Jeng SF, Lai JS, Cheng CK, Hu MH (1997) The relation between standing balance and walking function in children with spastic diplegic cerebral palsy. Dev Med Child Neurol 39:106112

Lin KC, Wu CY, Trombly CA (1998) Effects of task goal on movement kinematics and line bisection performance in adults without disabilities. Am J Occup Ther 52:179-187

Lipsitz LA (2002) Dynamics of stability: the physiologic basis of functional health and frailty. J Gerontol A Biol Sci Med Sci 57:B115-B125

McNevin NH, Wulf G (2002) Attentional focus on supra-postural tasks affects postural control. Hum Mov Sci 21:187-202

McNevin NH, Shea CH, Wulf G (2003) Increasing the distance of an external focus of attention enhances learning. Psychol Res 67:22-29

Nashner LM, Shumway-Cook A, Marin O (1983) Stance posture control in select groups of children with cerebral palsy: deficits in sensory organization and muscular coordination. Exp Brain Res 49:393-409

Newell KM, Slobounov SM, Slobounova ES, Molenaar PC (1997) Stochastic processes in postural center-of-pressure profiles. Exp Brain Res 113:158-164
Nichols DS (1997) Balance retraining after stroke using force platform biofeedback. Phys Ther 77:553-558

Pincus SM (1991) Approximate entropy as a measure of system complexity. Proc Natl Acad Sci USA 88:2297-2301

Prieto TE, Myklebust JB, Hoffmann RG, Lovett EG, Myklebust BM (1996) Measures of postural steadiness: differences between healthy young and elderly adults. IEEE Trans Biomed Eng 43:956-966

Raymakers JA, Samson MM, Verhaar HJJ (2005) The assessment of body sway and the choice of the stability parameter(s). Gait Posture 21:48-58

Richman JS, Moorman JR (2000) Physiological time-series analysis using approximate entropy and sample entropy. Am J Physiol Heart Circ Physiol 278:H2039-H2049

Riley MA, Balasubramaniam R, Turvey MT (1999) Recurrence quantification analysis of postural fluctuations. Gait Posture 9:65-78

Roerdink M, De Haart M, Daffertshofer A, Donker SF, Geurts AC, Beek PJ (2006) Dynamical structure of center-of-pressure trajectories in patients recovering from stroke. Exp Brain Res 174:256-269

Rose J, Wolff DR, Jones VK, Bloch DA, Oehlert JW, Gamble JG (2002) Postural balance in children with cerebral palsy. Dev Med Child Neurol 44:58-63

Rougier P (2003) Visual feedback induces opposite effects on elementary centre of gravity and centre of pressure minus centre of gravity motions in undisturbed upright stance. Clin Biomech 18:341-349

Schmit JM, Riley MA, Dalvi A, Sahay A, Shear PK, Shockley KD, Pun RY (2006) Deterministic center of pressure patterns characterize postural instability in Parkinson's disease. Exp Brain Res 168:357-367

Stackhouse C, Shewokis PA, Pierce SR, Smith B, McCarthy J, Tucker C (2007) Gait initiation in children with cerebral palsy. Gait Posture 26:301-308

Theiler J, Eubank S, Longtin A, Galdrikian B, Farmer JD (1992) Testing for nonlinearity in time series: the method of surrogate data. Physica D 58:77-94

Van der Weel FR, van der Meer ALH, Lee DN (1991) Effect of task on movement control in cerebral palsy: implications for assessment and therapy. Dev Med Child Neurol 33:419-426

Volman MJ, Wijnroks A, Vermeer A (2002) Effect of task context on reaching performance in children with spastic hemiparesis. Clin Rehabil 16:684-692

Woollacott MH, Burtner P (1996) Neural and musculoskeletal contributions to the development of stance balance control in typical children and in children with cerebral palsy. Acta Paediatr Suppl 416:58-62

Woollacott M, Shumway-Cook A (2002) Attention and the control of posture and gait: a review of an emerging area of research. Gait Posture 16:1-14

Wulf G, Prinz W (2001) Directing attention to movement effects enhances learning: a review. Psychon Bull Rev 8:648-660

Wulf G, McNevin N, Shea CH (2001) The automaticity of complex motor skill learning as a function of attentional focus. Q J Exp Psychol A 54:1143-1154 